\title{
Research Paper: Patient Satisfaction With Pre-hospital Emergency Care in Qom Province in 2017
}

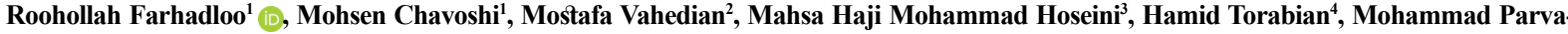 \\ resh-Masoud $^{5^{*}}$ (D, Mohammad Reza Azadeh ${ }^{6}$ \\ 1. Emergency Medical Center of Qom, Qom University of Medical Sciences, Qom, Iran. \\ 2. Clinical Research and Development Center, Qom University of Medical Sciences, Qom, Iran. \\ 3. Department of Emergency, Faculty of Paramedical, Qom University of Medical Sciences, Qom, Iran. \\ 4. Department of Pediatric Nursing, Faculty of Nursing and Midwifery, Qom University of Medical Science and Health Service, Qom, Iran \\ 5. School of Nursing and Midwifery, Tehran University of Medical Sciences, Tehran, Iran. \\ 6. School of Nursing and Midwifery, Qom University of Medical Science, Qom, Iran.
}

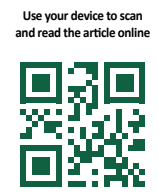

Citation: Farhadloo R, Chavoshi M, Vahedian M, Haji Mohammad Hoseini M, Torabian H, Parvaresh-Masoud M, Azadeh MR Patient Satisfaction With Pre-hospital Emergency Care in Qom Province in 2017. Health in Emergencies and Disasters Quarterly. 2019; 5 (2) :99-104. http://dx.doi.org/10.32598/hdq.5.2.199.4

http://dx.doi.org/10.32598/hdq.5.2.199.4

\section{(c) (i) (5)}

Article info:

Received: 18 May 2019

Accepted: 11 Nov 2019

Available Online: 01 Jan 2020

\section{Keywords:}

Satisfaction, Emergency medical services, Pre-hospital emergency

\section{ABSTRACT}

Background: Pre-hospital emergency is usually the first phase of treatment. The most serious emergency cases before any action in health care unit is to know what services are the cause of satisfaction or dissatisfaction of the patients with the overall emergency care. The aim of this study was to evaluate the satisfaction of recipients of pre-hospital emergency care in Qom City.

Materials and Methods: In this descriptive study 400 patients who transported to the hospitals by Qom EMS were selected by using simple random sampling. Data were collected by using of satisfaction questionnaire. The data gathered through telephone interviews. Data were collected by using a demographic questionnaire and a Consumer Emergency Care Satisfaction Scale (CECSS) and then analyzed by SPSS v. 16 software using descriptive and Chi-square tests.

Results: The results showed that the satisfaction rate from emergency ambulances and the efficiency of the emergency staff was moderate, and the satisfaction from technicians, the rate of acting professionally, performance of technicians, and the general opinion of patients were high. Also, the highest level of satisfaction (70\%) belonged to technicians and the least satisfaction $(26 \%)$ belonged to ambulance.

Conclusion: Although the satisfaction rate of Qom pre-hospital emergency staff related to the performance of ambulance was low due to some shortcomings and deficiencies;however the overall rate of satisfaction from pre-hospital emergency care in Qom City was at a good and acceptable level.

\section{* Corresponding Author: \\ Mohammad Parvaresh Masoud, PhD.}

Address: School of Nursing and Midwifery, Tehran University of Medical Sciences, Tehran, Iran.

E-mail: $m$ parvaresh@razi.tums.ac.ir 


\section{Introduction}

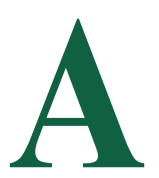

$\mathrm{n}$ indicator for investigating the effectiveness of the quality of services and care in standard hospital accreditation is patient satisfaction [1]. The satisfaction of the patient with the services provided is among the main goals of the healthcare system.

Accordingly, it could indicate the degree of adaptation of appropriate quality and quantity of services in their viewpoints [2]. Satisfaction is a concept with particular importance in medical care and considered a worthwhile purpose in patient care [3].

Patient satisfaction is an essential component of assessing the health status of the community and an indicator of measuring the quality of healthcare services. Besides, it could be influenced by the understanding and interpretation of events and clinical conditions by individuals $[4,5]$. An essential criterion for community development is providing healthcare services to the public. Moreover, the most crucial part of healthcare services is probably emergency care, especially at the pre-hospital admission level [6]. In other words, how services are provided in the emergency department represents the overall condition of services in society.

The quality of services provided in management centers and emergency medical care centers significantly reduces mortality before referring patients to the hospital and on the scene. Improving the quality of services in medical emergencies section requires an accurate understanding of the status quo and examining the existing problems $[7,8]$.

The pre-hospital emergency is a community-based health management system coordinated with the entire healthcare system. Emergency medical centers are among the major pillars of medical services worldwide. The main goal of this system is to provide satisfactory services in the shortest possible time following world-class scientific standards [9]. In healthcare systems, the first encounter with critical and emergency patients is usually with prehospital emergencies. The satisfaction of patients with this system could also affect their contentment with other areas [10]. The most frequent factors affecting patient satisfaction include mental perception, patients' awareness about their rights, staff's mental-related communication skills with patients, as well as, and demographic characteristics such as age, gender, educational level, personality traits, and cultural and socioeconomic status [11].

In the study by Vardanjani (2014), the highest and lowest satisfaction levels were related to pre-hospital emergency performance, and the performance of technicians, respectively [10]. In the study by Willis (2015) , the study participants expressed their satisfaction with the structure and process of the services provided. Despite overall satisfaction, dimensions, such as structure, the sense of comfort in the ambulance, and the response time process have led to a decreased level of satisfaction [12]. This study aimed to evaluate the satisfaction level of pre-hospital emergency bases among service recipients in Qom City, Iran, in 2017.

\section{Materials and Methods}

This cross-sectional descriptive study aimed to evaluate the satisfaction level of service recipients with the pre-hospital emergency in Qom City, Iran, in 2017. It was conducted in the areas covered by Qom University of Medical Sciences. Among those who called the Qom emergency department, 420 patients were included in the study. The study subjects were patients for whom an ambulance was sent, received medical services on the scene, and was eventually taken and admitted to the hospital. The study sample characteristics were recorded in the Pre-hospital Care Report (PCR) form. The forms were selected using a simple random sampling method; they were selected among all the forms obtained in 2017 in the list of patients transferred to Qom medical centers by the Emergency Department. The data collection was conducted based on a telephone interview. If the subject did not respond to the call due to a low level of literacy or lack of knowledge of Persian language or similar reasons, similar interviews were conducted with other family members or patient caregivers; otherwise, the subject was excluded from the study.

According to Esmaeili et al., the number of samples required for the study was calculated to be 384 , based on the criteria, such as $\mathrm{P}=0.5,95 \%$ confidence interval, and $5 \%$ accuracy [10]. While taking the possible exclusion of the samples into account, 400 possible PCR forms were investigated in this study. The collected information was analyzed in SPSS using descriptive analysis and the Chi-squared test.

The necessary data were collected using a demographic questionnaire (consisting of 6 questions) and a Consumer Emergency Care Satisfaction Scale (CECSS). The demographic questionnaire included factors, such as age, gender, educational level, disease type, place of residence, and previous contact history. The CECSS included 6 areas of satisfaction assessment, including ambulance, technician behavior, technician professionalism, technician performance, emergency department perfor- 
mance on the scene, and the overall opinion of patients about the emergency department ( $\mathrm{N}=24$ questions). The questionnaire's validity was explored and confirmed in a similar study by Esmaeili et al. The questionnaire's reliability was measured as 0.81 based on Cronbach's alpha coefficient by Esmaeili and associates [10].

Scoring was based on a Likert-type scale (very good, good, medium, weak) and the obtainable scores varied between 1 and 4 . The first four items, including ambulance, technician behavior, technician professionalism, technician performance had a minimum score of 4 and a maximum score of 20 (scores 4-9 indicated a low, 10-15: average, \& 16-20: a high level of satisfaction). For the "emergency performance" item, the obtainable score varied between 3 and 12 (scores 3-6: low, 7-9: average, \& 10-12: a high level of satisfaction). For the "overall opinion of patient" item, the achievable score varied between 1 and 3 (score 1: weak, 2: average, \& 3: a high level of satisfaction).

Mean \pm SD statistics were used to describe quantitative data, and frequency and percentage were used for presenting the nominal and ordinal data. The Chi-squared test was used to investigate the relationship between independent variables and satisfaction ranges. $\mathrm{P}<0.05$ was considered as the significance level of the tests.

\section{Results}

In this study, a total of 400 patients were investigated. They were transferred to the hospital after a telephone call to the emergency department of Qom City, Iran.

Table 1. Frequency and percentage of participants in terms of demographic variables

\begin{tabular}{ccc}
\hline Variables & Frequency & No. (\%) \\
\hline \multirow{2}{*}{ Age $(\mathrm{y})$} & $1-30$ years & $88(22)$ \\
& $31-60$ years & $208(52)$ \\
Gender & $61-94$ years & $104(26)$ \\
& Male & $202(50.5)$ \\
Level of education & Female & $198(49.5)$ \\
& Below diploma & $202(55)$ \\
& Diploma & $100(25)$ \\
Number of calls & Above diploma & $80(20)$ \\
& $1-3$ calls & $300(75)$ \\
& $4-7$ calls & $68(17)$ \\
& $7-10$ calls & $32(32)$ \\
& Cardiac disease & $100(25)$ \\
Type of disease & Stroke & $18(4.5)$ \\
& Digestive tract disease & $28(7)$ \\
& Diabetes & $18(4.5)$
\end{tabular}

Table 2. The relationship between areas of satisfaction based on the underlying variables

\begin{tabular}{|c|c|c|c|c|c|c|c|c|c|c|c|c|}
\hline \multirow{2}{*}{ Variables } & \multicolumn{2}{|c|}{ Ambulance } & \multicolumn{2}{|c|}{ Technician } & \multicolumn{2}{|c|}{ Skill } & \multicolumn{2}{|c|}{ Performance } & \multicolumn{2}{|c|}{ Effectiveness } & \multicolumn{2}{|c|}{ Overall Opinion } \\
\hline & $x^{2}$ & $\mathbf{P}$ & $x^{2}$ & $\mathbf{P}$ & $x^{2}$ & $\mathbf{P}$ & $x^{2}$ & $\mathbf{P}$ & $x^{2}$ & $\mathbf{P}$ & $x^{2}$ & $\mathbf{P}$ \\
\hline Gender & 6.08 & 0.048 & 3.20 & 0.20 & 4.30 & 0.12 & 0.57 & 0.75 & 6.40 & 0.04 & 2.10 & 0.35 \\
\hline Education & 9.70 & 0.046 & 3.50 & 0.48 & 6.05 & 0.20 & 2.90 & 0.57 & 1.80 & 0.7 & 1.50 & 0.80 \\
\hline Age (y) & 0.83 & 0.66 & 0.86 & 0.51 & 0.45 & 0.80 & 3 & 0.22 & 1.74 & 0.42 & 0.16 & 0.92 \\
\hline Previous contact history & 1.90 & 0.02 & 3.35 & 0.50 & 6.10 & 0.20 & 7.30 & 0.12 & 7.50 & 0.11 & 4.60 & 0.33 \\
\hline
\end{tabular}


Table 3. Determining the level of satisfaction of emergency treatment services in recipients based on independent variables

\begin{tabular}{|c|c|c|c|}
\hline \multirow{2}{*}{ Variables } & \multicolumn{3}{|c|}{ No. (\%) } \\
\hline & Low & Medium & High \\
\hline Ambulance & $136(34)$ & $160(40)$ & $104(26)$ \\
\hline Technician behavior & $16(4)$ & $104(26)$ & $280(70)$ \\
\hline Skill & $20(5)$ & $116(29)$ & $264(66)$ \\
\hline Performance & $16(4)$ & $164(41)$ & $220(55)$ \\
\hline Effectiveness & $60(15)$ & $216(54)$ & $124(31)$ \\
\hline Overall opinion & $16(4)$ & $156(34)$ & $228(62)$ \\
\hline
\end{tabular}

The Mean \pm SD age of the participants was 47.3 (20.40) years, with a minimum of 1 and a maximum of 94 years. In terms of age variable, $88(22 \%)$ participants were in the age range of $1-30 ; 208(52 \%)$ participants were 31 60 years, and $104(26 \%)$ participants were 61-94. Furthermore, $202(50.5 \%)$ participants were male and 198 $(49.5 \%)$ were female. The highest rate of contact belonged to the patients with an educational level of below diploma with 220 calls; this rate was followed by those subjects with diploma degree with 100 calls. The lowest contact rate belonged to the patients with an educational level of above diploma degree with 80 calls. The frequency of 1-3 calls equaled 300 (75\%), 4-7 was 68 $(17 \%)$, and 7-10 was measured as $32(32 \%)$.

The Mean \pm SD number of calls was 2.7 (2.40) times; the minimum number was 1 and the maximum was 10 times. The obtained results suggested the following data regarding disease type among those who called the emergency department. The patients with cardiovascular disease had the maximum number of calls with 100 (25\%) calls and those with obstetrics and gynecology diseases had the lowest number of calls with $10(2.5 \%)$ calls (Table 1$)$.

Table 2 lists the relationship between the underlying variables and different areas of satisfaction with emergency services. Table 2 indicates that the satisfaction of patients with the ambulance was statistically significant. These data were based on the variables, such as gender $(\mathrm{P}=0.048)$, educational level $(\mathrm{P}=0.046)$, and previous contact history $(\mathrm{P}=0.02)$. Satisfaction with emergency performance was also significant concerning the gender variable $(\mathrm{P}=0.04)$ (Table 2$)$.

The satisfaction level of emergency treatment recipients based on the independent variables revealed that the highest level of satisfaction was related to the technician behav- ior $(70 \%)$, and the lowest level belonged to the ambulance (26\%). Overall, $4 \%$ of the explored samples had low satisfaction, 34\% had moderate satisfaction, and $62 \%$ had a high satisfaction level with emergency services (Table 3 ).

\section{Discussion}

The present study results indicated that the investigated patients were satisfied with the emergency services provided to them by the Qom emergency department. A large extent of this satisfaction related to the performance of emergency technicians; however, low rates were reported for the satisfaction with the ambulance. In line with the findings of the present study, research by Johansson et al. in Sweden suggested favorable levels of satisfaction of patients with pre-hospital emergency services [13]. Various domestic and international studies also indicated overall satisfaction with the desired level of emergency services [10, 12, 14-17].

Investigating patient satisfaction based on the questionnaire items revealed that satisfaction with emergency ambulances and emergency staff efficiency was at a medium level; satisfaction with the technician behavior, technician professionalism, technician performance, and overall opinion of patients about emergency was at a high level. Moreover, the highest level of satisfaction related to technician behavior (70\%) and the lowest level of satisfaction belonged to the ambulance (26\%).

In line with the present study, the study by Dahmardehei indicated that $80 \%$ of patients who referred to the Zahedan emergency department presented an overall satisfaction with the emergency services. The highest level of satisfaction related to professionalism and the behavior of technicians with patients and the lowest level belonged to the ambulance [16]. The present study 
also suggested a significant relationship between patient satisfaction and professionalism, self-confidence, and employee competence. In the study by Jadidi, the lowest level of satisfaction related to the efficiency area of emergency technicians [14]. In the study of Kuisma et al., a lack of high efficiency of staff in patient problem management and failure to establish proper communication with the patient by emergency personnel were the main factors in reducing the satisfaction level with the emergency services [18]. Inconsistent with the present study, Vardanjani et al. reported that the lowest satisfaction level related to the performance of technicians; the satisfaction level with the ambulance was optimal [10]. Some international studies also indicated that patients were satisfied with ambulance transfers [19-21].

High satisfaction level with the professionalism and behavior of technicians of Qom emergency department could be attributed to the change in the recruitment process of emergency staff in recent years; continuous in-service training and in-service tests during working years; performing numerous missions, and gaining much experience in the field of practice along with scientific training. According to Vaitkaitis, nurses are powerful and competent if having adequate knowledge and skills and be able to appropriately use this information [22].

Moreover, the present study results indicated that the staff of Qom emergency department bases have appropriate and acceptable sympathy and goodwill in managing patients in terms of technician behavior. However, the lack of modern emergency ambulances, the inadequacy of medical equipment, as well as the unsuitable space inside the ambulances reduced the satisfaction of emergency treatment recipients concerning satisfaction with the ambulance. In line with the present study, Dahmardehei also argued that patients were less satisfied with ambulances and medical equipment [16]. Accordingly, it is necessary to address the exhaustion of ambulances by replacing new and modern ambulances. Besides, resolving the problems regarding equipment shortages of emergency ambulances is of significant importance. Most studies have revealed that patient satisfaction is a useful indicator for estimating the quality of medical care $[12,14,15,23,24]$.

Nevertheless, it is essential to evaluate this satisfaction precisely. Perhaps the most critical and challenging step in evaluating the satisfaction level is to prepare a research questionnaire. The difference in the structure of questionnaires is the reason for the discrepancies in the results of studies conducted on satisfaction. Considering the disease type and other factors that may affect the patient's satisfaction, it is reasonable to assume that patients' expectations of emergency care are different. To increase the knowledge about patient satisfaction with emergency care, further in-depth studies, like qualitative studies, are required for patients to better explain which services they are satisfied with and in what quality [15].

\section{Conclusion}

The current study data suggested that patients' satisfaction with pre-hospital emergency services in Qom was at a reasonable and satisfactory level. However, the satisfaction level with the Qom emergency department staff was low in terms of satisfaction with the ambulance. This finding highlights the shortcomings in this section. However, the proper performance of employees in managing patients and the professionalism of employees have overshadowed this weakness. The pre-hospital emergency is among the most essential and foremost areas of the treatment system in each society. The relevant authorities should consider the weaknesses in this sector as an essential and effective priority. Due to the shortcomings in emergency department ambulances, especially in terms of patient comfort and safety to transfer trauma patients, obtaining modern ambulances is a reasonable request of patients and their companions. Moreover, due to the low number of studies conducted on satisfaction with the emergency department of the country, it seems necessary to conduct more studies in this field.

\section{Ethical Considerations}

\section{Compliance with ethical guidelines}

This study was approved by the Research Council and the Research Ethics Committee of Qom University of Medical Sciences (Code: IR.MUQ.REC.1397.098).

\section{Funding}

The present article is the result of a research project No. 97943, and financially supported by the Vice-Chancellor for Research and Technology of Qom University of Medical Sciences.

\section{Authors' contributions}

All authors contributed in preparing this article.

Conflict of interest

The authors declared no conflict of interest. 


\section{Acknowledgments}

The authors hereby express their gratitude towards all study participants and the Vice-Chancellor for Research and Technology of Qom University of Medical Sciences for funding this project. The authors also thank all the pre-hospital emergency staff for their efforts to provide the best services despite the limitations.

\section{References}

[1] Kolivand P, Ali Hakami M, Eghbali F, Monjamed Z, Sharifi F. Patient satisfaction of the quality of patient teaching in Khatam Alanbia hospital. The Neuroscience Journal of Shefaye Khatam. 2015; 3(3):25-31. [DOI:10.18869/acadpub.shefa.3.3.25]

[2] Roberts M, Hsiao W, Berman P, Reich M. Getting health reform right: a guide to improving performance and equity. Oxford: Oxford Scholarship Online; 2008. [DOI:10.1093/acprof:o so/9780195371505.001.0001]

[3] Azizi F, Hanifi N, Rabie Siahkali S, Haghani S. [Investigating the factors affecting the triple dimensions of patients' satisfaction of those referred to the emergency department in Zanjan, Iran in 2017 (Persian)]. Journal of Military Medicine. 2018; 20(5):538-45.

[4] Khursheed M, Fayyaz J, Zia N, Feroze A, Jamil A, Baqir SM. Real-time patient satisfaction of emergency department services in a tertiary-care hospital in Karachi, Pakistan. Emergency Medicine: Open Access. 2014; 04(03). [DOI:10.4172/2165-7548.1000188]

[5] Datobar H, Alijanpour S, Khafri S, Jahani M, Naderi R. [Patient's Satisfaction of emergency department affiliated hospital of Babol University of Medical Sciences in 2013-2014 (Persian)]. Journal of Babol University Of Medical Sciences. 2016; 18(4):56-62. http:// jbums.org/article-1-5626-fa.pdf

[6] Ali J, Mahdi S, Behrooz I, Mahdi H. [Level of patient's satisfaction from emergency medical services in Markazi Province: A Cross sectional Study (Persian)]. Iranian Journal of Emergency Medicine. 2016; 3(2):58-65.

[7] Mistovich JJ, Hafen BQ, Karren KJ, Werman HA, Hafen B. Prehospital emergency care. Howard A. Werman, editor. Upper Saddle River, N.J.: Brady Prentice Hall Health; 2004.

[8] Willis S, Dalrymple R. Fundamentals of paramedic practice: A systems approach. $2^{\text {nd }}$ edition. New Jersey: John Wiley \& Sons; 2015.

[9] Saberi Nia A, Nekouei Moghadam Mahmoudi Meymand F. [Identify stressful factors causing dissatisfaction in pre-hospital emergency personnel in Kerman (Persian)]. Payavard Salamat. 2013; 6(6):489-97.

[10] Esmaeili Vardanjani A, Moghadasi J, Rafiei H, Masoudi R, Mohammadi M, Lalegani $\mathrm{H}$, et al. [Satisfaction from pre-hospital emergency medical services: A cross sectional study in Shahrekord (Persian)]. Journal Of Clinical Research In Paramedical Sciences. 2014; 3(2):103-13. https://sites.kowsarpub.com/jcrps/ articles/82096.html
[11] Khezri L, Ravanipour M, Jahanpour F, Mirzaee K. [Assessment of patient satisfaction with nursing services provided in general wards of university hospitals in Bushehr 2012 (Persian)]. Nursing Journal of the Vulnerable. 2015; 2(3):36-46.

[12] Dantas RAN, Dantas DV, Nascimento JCP, Sarmento SDG, Oliveira SPD. Evaluation of satisfaction of users assisted by prehospital mobile emergency care service. Saúde em Debate. 2018; 42(116):191-202. [DOI:10.1590/0103-1104201811615]

[13] Johansson A, Ekwall A, Wihlborg J. Patient satisfaction with ambulance care services: Survey from two districts in southern Sweden. International Emergency Nursing. 2011; 19(2):86-9. [DOI:10.1016/j.ienj.2010.03.002] [PMID]

[14] Jadidi A, Safarabadi M, Irannejad B, Harorani M. [Level of patients' satisfaction from emergency medical services in Markazi province: A cross sectional study (Persian)]. Iranian Journal of Emergency Medicine. 2016; 3(2):58-65.

[15] Larsson G, Strömberg U, Rogmark C, Nilsdotter A. Patient satisfaction with pre-hospital emergency care following a hip fracture: A prospective questionnaire-based study. BMC Nursing. 2018; 17(1):38. [DOI:10.1186/s12912-018-0307-x] [PMID] [PMCID]

[16] Dahmardehei M, Rashedi F, Koosha F, Khosh Sima S. [A study on satisfaction level of patients referred to emergency centers of Zahedan (Persian)]. Journal of Zabol University of Medical Sciences and Health Services. 2014; 6(1):60-7.

[17] Blomstedt K, Nilsson H, Johansson A. The public's perception of pre-hospital emergency care in the County of Skane, southern Sweden. International Emergency Nursing. 2013; 21(2):136-42. [DOI:10.1016/j.ienj.2012.05.004] [PMID]

[18] Kuisma M, Määttä T, Hakala T, Sivula T, Nousila-Wiik M. Customer satisfaction measurement in emergency medical services. Academic Emergency Medicine. 2003; 10(7):812-5. [DOI:10.1197/ aemj.10.7.812] [PMID]

[19] Swain AH, Al-Salami M, Hoyle SR, Larsen PD. Patient satisfaction and outcome using emergency care practitioners in New Zealand. Emergency Medicine Australasia. 2012; 24(2):175-80. [DOI:10.1111/j.1742-6723.2011.01525.x] [PMID]

[20] Keene T, Davis M, Brook C. Characteristics and outcomes of patients assessed by paramedics and not transported to hospital: A pilot study. Australasian Journal of Paramedicine. 2015; 12(2). [DOI:10.33151/ajp.12.2.231]

[21] Soriano Clemor C, Cano FS, Gimeno FM. [Quality analysis by external users of the non-medical health transport unit of Alicante (Spanish)]. 2011; 26(1):12-7. [DOI:10.1016/j. cali.2010.08.001] [PMID]

[22] Vaitkaitis D. EMS systems in Lithuania. Resuscitation. 2008; 76(3):329-32. [DOI:10.1016/j.resuscitation.2007.07.028] [PMID]

[23] Batbaatar E, Dorjdagva J, Luvsannyam A, Savino MM, Amenta PJPiph. Determinants of patient satisfaction: a systematic review. 2017; 137(2):89-101. [DOI:10.1177/1757913916634136] [PMID]

[24] Messina G, Vencia F, Mecheroni S, Dionisi S, Baragatti L, Nante N. Factors affecting patient satisfaction with emergency department care: An Italian rural hospital. Global Journal of Health Science. 2014; 7(4):30-9. [DOI:10.5539/gihs.v7n4p30] [PMID] [PMCID] 